\title{
COMUNICACIÓN Y TECNOPOLÍTICA EN EL ECUADOR FACEBOOK: NUEVA HERRAMIENTA DE INTERACCIÓN Y COMUNICACIÓN POLÍTICA
}

\author{
COMMUNICATION AND \\ TECHNOPOLITICS IN ECUADOR \\ FACEBOOK: NEW INTERACTION TOOL AND \\ POLITICAL COMMUNICATION
}

ALBERTO RENGIFO HIDALGO ${ }^{1}$

Recibido: 10 de enero de 2017

Aceptado: 6 de marzo de 2017

${ }^{1}$ Universidad Andina Simón Bolívar -Sede Ecuador-, Área de Comunicación, Quito, Ecuador (darh_mj@ hotmail.com). 



\title{
COMUNICACIÓN Y \\ TECNOPOLÍTICA EN EL ECUADOR FACEBOOK: NUEVA HERRAMIENTA DE INTERACCIÓN Y COMUNICACIÓN POLÍTICA
}

\author{
COMMUNICATION AND \\ TECHNOPOLITICS IN ECUADOR \\ FACEBOOK: NEW INTERACTION TOOL AND \\ POLITICAL COMMUNICATION
}

ALBERTO RENGIFO HIDALGO

PALABRAS CLAVE: tecnopolítica, redes sociales, Facebook, actoría política, procesos electorales.

KEY WORDS: technopolitics, Social networks, Facebook, political participation, electoral processes.

\section{RESUMEN}

En los últimos años, las redes sociales se han convertido en herramientas y espacios comunicacionales para la interacción político-mediática gracias a su facultad para la interrelación con las audiencias. En el Ecuador los espacios de hacer política han cambiado desde la plaza pública, el debate televisado, a la confrontación de "shares" y "likes" en las redes sociales. Esta nueva plaza pública digital ha permitido que la relación de poder entre gobernantes y gobernados sea menos vertical a medida que los gobernados -la sociedad 
en su conjunto- han tenido acceso a mejores tecnologías para comunicarse y organizarse entre ellos. Precisamente esta renovación política a través de la tecnología social -Tecnopolítica- ha permitido que emerja un nuevo modelo de hacer las cosas, de comunicarse con los votantes y de hacer política. Esta investigación es un análisis cuantitativo que visibiliza cómo Facebook se ha convertido en la fuente de información privilegiada para la toma de decisiones políticas y un espacio de actoría de los habitantes de Quito.

\section{ABSTRACT}

In the past few years, social media have become a relevant tool and an important communicative space for political-high profile interaction due to its potential for enhancing audience exchange. In Ecuador the places to do politics are changing from the traditional mass media spaces, such as TV debates, to the digital ones like Facebook with likes and shares. These new digital spaces allow that the relation among political leaders and civil society is less vertical (top-down) to the extent that these latest have had more access to new technologies. The social media has created a new way-"Technopolitics" or social technology- to communicate and do politics. The current proposal consists on a quantitative analysis that visualizes the way Facebook has become a primary source of information for political decision-making and a space for participating among young people in Quito.

\section{INTRODUCCIÓN}

La comunicación y todos sus procesos y evoluciones comunicativas se han convertido en un elemento vital y en un eje transversal en la vida del ser humano, ya que sin estos no se podría establecer relaciones con el entorno.

En la actualidad-aparte de los medios de comunicación tradicionales como la prensa impresa, la televisión o la radio- prima la conectividad a la red (internet) y hay una dependencia hacia los "aparatos" (smarthphones y computadoras) que posibilitan este acceso. Podríamos decir que vivimos en una Aldea Global, como lo manifestó McLuhan, en una sociedad en la que todos estamos 
comunicados mediante las herramientas tecnológicas.

Vivimos en un estado de la híperconectividad; "sin conectividad, hoy es imposible competir. No hay organizaciones grandes, medianas o pequeñas que no tengan esto muy en cuenta. Las personas saben que no estar conectado es altamente riesgoso para su desempeño social y laboral" (Igarza Roberto, 2009, p. 20). Es importante aclarar que no es malo estar en "hiperconectividad", inclusive, Manuel Castells, en su libro La era de la información, planteó que vivimos con los medios y junto a los medios; es más, sería algo descabellado plantear la idea de que en nuestra época no usemos la tecnología o pretendamos desconectarnos del entorno.
Precisamente, esta "conectividad" de la sociedad ha hecho que, en los últimos años, las redes sociales se posicionen en la palestra de la información y comunicación y se conviertan en herramientas y fuentes de información privilegiada en lo que corresponde a la toma de decisiones políticas y a su actoría en los jóvenes. Es decir, que estas herramientas han ganado un espacio importante en las relaciones de interacción política del electorado juvenil.

Ahora bien, la pregunta que se genera es si en el Ecuador los espacios de hacer política han cambiado desde la plaza pública, el debate televisado, a la confrontación de "shares" y "likes" en redes sociales.

\section{REDES SOCIALES, NUEVAS HERRAMIENTAS DE INTERACCIÓN Y COMUNICACIÓN POLÍTICA}

Las redes sociales -Facebook principalmente- son herramientas que, si bien rompen con esa relación de comunicación cara a cara en espacios de co-presencia, según Thompson (1998), se han transformado en redes de sociabilidad; es decir, "ambientes" en donde puedes interactuar y aportar a una conversación, debate, clase, construcción de conceptos al instante, haciendo que los "límites" o barreras geográficas aparentemente desaparezcan.

Estas redes, así como las demandas de la sociedad, han ido evolucionando a través de los años. Por ejemplo, en 1995 la red de mayor uso y popularidad fue classmates.com, en el 2002 friendster, en 2003 MySpace, en el 2004 Hi5 y Facebook, hasta llegar a la actualidad en donde, según un es- 
tudio realizado por Martínez, Fortuño y Vidal (2016) "Facebook y Twitter son de las redes sociales más utilizadas a nivel mundial, siendo Facebook la red social líder a nivel de uso personal, académico e informativo" (p. 21).

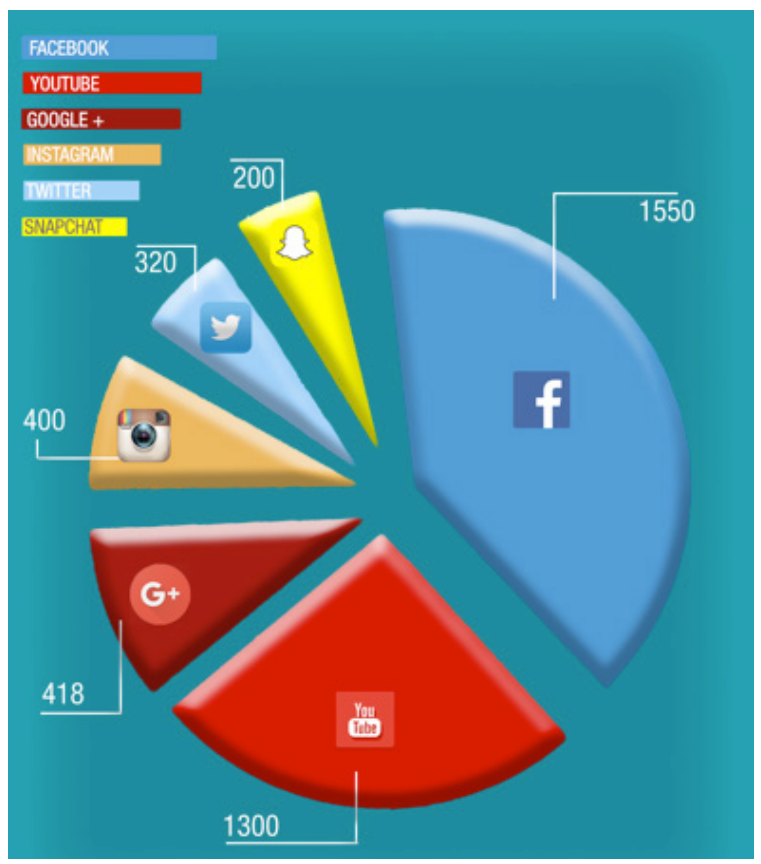

Fig. 1. Redes sociales más usadas a nivel mundial, 2016.

Fuente: Multiplicalia.com

Vivimos entonces en un espacio de comunicación diferente al de hace 10 años, la sociedad consume información a un ritmo jamás antes visto; nunca antes se ha leído tanto como se lee ahora, ya no existen distancias comunicacionales, la interacción es inmediata.
Según datos de la agencia global de comunicación y consultoría con presencia en más de 11 países, We Are Social, en enero de 2016 de los más de 7.395 millones de habitantes del planeta, 3.419 millones tienen acceso a internet (un incremento del $10 \%$ en un año) 
y 2.307.000.000 usan regularmente las redes sociales ( $+10 \%$ desde enero de 2015), 3.790.000.000 personas utilizan un teléfono móvil (+ 4\% en un año) y 1.968 millones de personas acceden a las redes sociales a través de estos. Los datos seguirán creciendo con una proyección anual del $15 \%$. Este irrefrenable crecimiento de la penetración de internet a nivel mundial, nos indica claramente que vivimos en una sociedad tecnologizada, en una sociedad-red que día a día se vuelve más dependiente de la comunicación e interacción en el ciberespacio.

Castells (2003) plantea que hubo un proceso de transición para llegar a este punto, ya que en la década del 70 inicia un surgimiento y constitución en el mundo de un paradigma tecnológico dominante gracias a la informática y las telecomunicaciones, hasta llegar a nuestra época, en donde la comunicación móvil y las redes sociales "juegan un papel fundamental, ya que se constituyen en un medio en donde cualquier contenido tiene cabida" (García-Galera y Valdivia, 2014, p.11) y en donde cada vez es más fácil informarse y participar al respecto, es decir que, como lo menciona García-Calera y Valdivia (2014), los límites del espacio y del tiempo se han borrado; los contenidos -todo tipo de contenido- están en la red y 2.400 millones de personas lo usan casi a diario.
Precisamente, la Internet se ha convertido en el instrumento clave y en el símbolo de este nuevo sistema tecnológico (Castells, 2007), en donde las nuevas plataformas de comunicación son, sin lugar a dudas, las redes sociales. Facebook, por ejemplo, ha marcado un hito en la forma de comunicación, en especial para los jóvenes, ya que no es solamente un medio para la difusión de eventos, sino que se crean acontecimientos -movilizaciones, participaciones políticas- solo por el hecho de difundirlos en las redes.

Esta incesante motivación por hacer de las redes sociales las nuevas plataformas de comunicación, interacción e información está fundamentada en el consumo informativo que realizan los jóvenes, ya que se relaciona con el deseo de defender sus puntos de vista y, sobre todo, entregar información a otros. Surge entonces la siguiente interrogante: ¿para qué y con qué finalidad son usadas estas redes sociales?

Para algunas personas las redes sociales son un espacio en donde no se genera conocimiento ni criticidad, un espacio en donde únicamente se "recepta" cualquier tipo de contenido sin un filtro de calidad académica e informativa; para otros, en cambio, las redes sociales se han transformado en un espacio privilegiado en donde los usuarios ya no desempeñan un único papel de recep- 
tores (lo que ocurre normalmente con los mass media tradicionales) sino que "asumen alternativamente el papel de receptores y el de emisores, alternancia casi innata a la comunicación interpersonal que ahora se traslada a la comunicación global, lo que aplicando a los medios de comunicación, ha hecho acuñar términos como -prosumidor- usuario que no es solo consumidor sino también creador de contenidos" (Del Hoyo, García y Fernández, 2014, p. 37).

Al convertirse en generadores y creadores de contenidos, los usuarios de las redes sociales, los prosumidores mediáticos, tienen una infinidad de aplicaciones de esta herramienta tecnológica, por lo que se apropian de esta y la transforman en una fuente privilegiada de información, comunicación y actoría política.

Esta evolución y crecimiento exponencial en el uso de estas nuevas herramientas de comunicación han cambiado la forma de actuar de muchas entidades, movimientos, empresas y personas, ya que los usuarios, otrora receptores de noticias, ya no se contentan con recibir la información dada por los medios tradicionales, ahora buscan ser los generadores de la misma y que esta sea más inmediata, más precisa, más confiable.

Esas tecnologías de la información han sido herramientas claras y espa- cios comunicacionales necesarios para la interacción político-mediática. Facebook es una de las redes más usadas por los jóvenes para la búsqueda de información y participación social. Según Scherman \& Arriagada (2010), esto se fundamenta en que "existe un cambio de proporciones en la manera de entender y ejercer la ciudadanía, ya que el compromiso y activismo político del público joven, ocurre mediante las tecnologías de información, que han creado y facilitado nuevas formas de comunicación y organización colectiva".

Para Acosta y Maya (2012), "movilizaciones sociales como Un millón de voces contra las Farc y la denominada Primavera árabe, que condujo a la caída de las dictaduras en Túnez y Egipto, son un claro ejemplo de cómo los jóvenes y Facebook se "unieron" y desempeñaron un papel protagónico. Es así como la Internet en general y las redes sociales en particular se configuran en un nuevo espacio público, en la nueva plaza pública para la participación y socialización de los sujetos en la era del ciberespacio" (p. 52).

Esta nueva plaza pública digital ha permitido que "la relación de poder entre gobernantes y gobernados sea menos vertical a medida que los gobernados - la sociedad en su conjunto- han tenido acceso a mejores tecnologías para comunicarse y organizarse entre 
ellos". Como lo plantea Antoni Gutiérrez Rubí (2104) existe un difuso, pero profundo, movimiento de renovación política a través de la tecnología social -Tecnopolítica- que ha permitido que emerja un nuevo modelo de hacer las cosas y de hacer política:

Dar primero la palabra, antes de pedir el voto. En una sociedad decepcionada, crítica y muy informada, la políti- ca está cada vez más vigilada por los ciudadanos; estos quieren poder decidir, o tener la posibilidad de hacerlo. Se multiplican las aplicaciones y plataformas que fiscalizan y monitorizan las actividades de los gobernantes, y algunas que permiten participar en política, comunicar necesidades y desacuerdos y tomar decisiones juntos (p. 14).

\section{LAS REDES SOCIALES, LA NUEVA "PLAZA” DE LA INTERACCIÓN POLÍTICA EN EL ECUADOR}

En el Ecuador el uso y apropiación de los espacios digitales, especialmente de Facebook, ha crecido notablemente. Según datos obtenidos mediante la Encuesta de Condiciones de Vida -ECVrealizada por el Instituto de Estadísticas y Censos del Ecuador, en el año 2015, cerca de un $98 \%$ de personas que están sobre la franja de edad de los 12 años tiene una cuenta en Facebook, y Quito es la ciudad con el mayor porcentaje de presencia en esta red social.

Estos datos nos permiten plantear la hipótesis de la existencia de una nueva plaza pública en la que existe una participación activa por parte de los ciudadanos, una participación en donde es "posible hacer más fácil la participación y la deliberación a gran escala, y, sobre todo, la capacidad de reconvertir a los militantes, simpatizantes o votantes en activistas" (Gutiérrez, 2014, p. 14). 


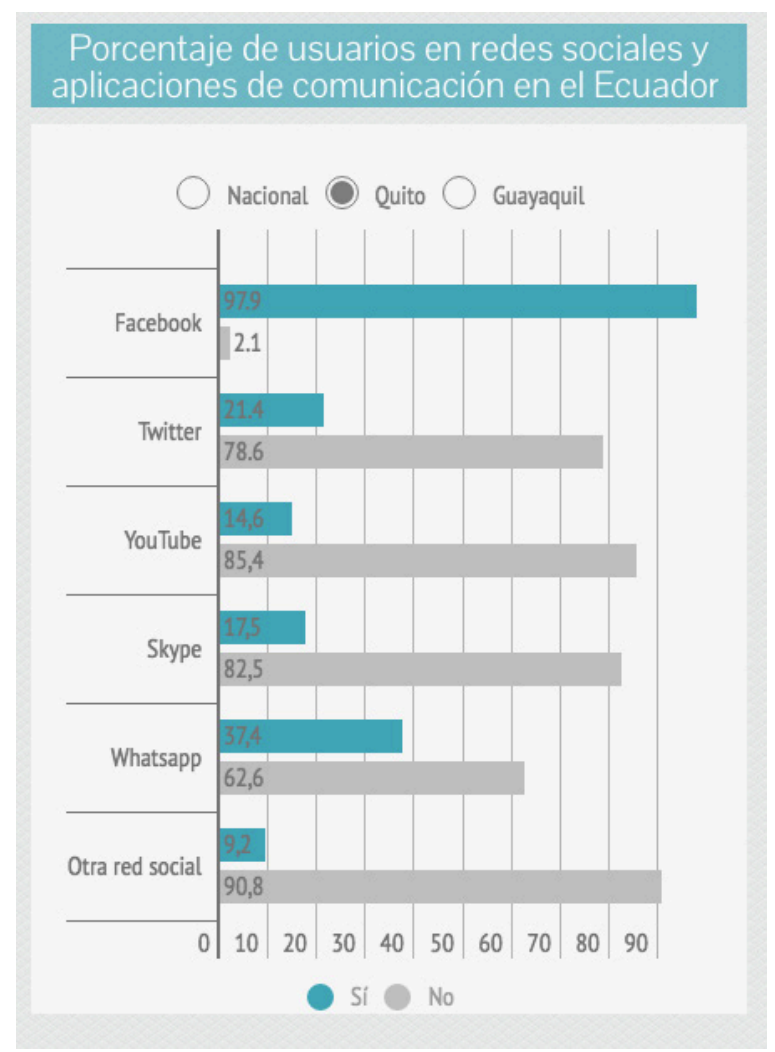

Fig. 2. Porcentaje de usuarios en redes sociales Ecuador.

Fuente: INEC, 2015.

Elaborado por Carla Sandoval, 2015. Diario “El Comercio".

Esta nueva plaza pública demanda más experticia, características y conocimientos por parte de los protagonistas, así como de los consumidores y prosumidores. Para el catedrático de Periodismo Digital, Christian Espinoza, la forma de comunicar, hacer y percibir la política en el Ecuador ha cambiado totalmente gracias a las redes sociales, "la política circulaba por las calles y hoy está atravesando por estas nuevas calles: las manifestaciones ya no requieren que sean concentraciones en lugares públicos o plazas. En esta nueva plaza, las manifestaciones son a diario, todos los días" (C. Espinoza, comunicación personal, 12 de septiembre de 2014). 
Nueva plaza pública que, sin lugar a dudas, se ha venido posicionando hasta convertirse en el espacio idóneo para que exista actoría política, ya que el plus que presenta frente a otros espacios es el de poder interactuar y convertir a sus usuarios en los protagonistas de la noticia, del evento, de la decisión. Esto ha hecho que los políticos también generen sus propias reglas con las cuales van marcando su agenda. Según Espinoza (2014), este "otro espacio" es la cohesión entre el político y el ciudadano:

Antes para acceder a los políticos, para comunicarse con ellos se debía pasar por muchos círculos, esos círculos hoy se han desinhibido, ahora hay una conexión directa con el ciudadano, y esto ha hecho que el nivel de influencia que tienen las personas con sus espacios propios, con sus publicaciones, sea cada vez más representativo.

Para Juan Carlos Vásconez, gerente de Ximah Digital, empresa de comunicación que tiene contratos con empresas privadas e instituciones públicas del Ecuador, las redes sociales, Facebook principalmente, son hoy por hoy la principal fuente informativa de la ciudadanía quiteña:
Facebook es muy bueno para llegar al público, es el canal donde existe mayor cantidad de usuarios y al mismo tiempo diversidad. Es una realidad que todo posicionamiento político, primero se lo busca en redes sociales, ya que al ser una fuente de acceso rápida, se transforma en un espacio privilegiado para la interacción social-política (J. Vásconez, comunicación personal, 20 de octubre de 2014).

Vásconez (2014) considera que la inmediatez, la opinión pública y el fácil acceso a la misma han hecho de Facebook una plataforma digital y mediática con información rápida y validada por el público, lo cual es el factor determinante para considerar a una plataforma como fuente de información privilegiada. Hoy en día, la opinión pública es la que manda. Los jóvenes tienen acceso a información que antes no la tenían, sus ideas son escuchadas y ellos son las masas y medidores de recepción en mensajes en redes sociales.

Por todo esto, podríamos establecer que Facebook es un espacio idóneo para la participación política, ya que se puede hacer conocer a algún personaje o alguna propuesta de forma masiva y efectiva. Al momento no se puede hablar a ciencia cierta de "obtener" una victoria electoral gracias a redes socia- 
les; sin embargo, hay que destacar que la "evolución" de los usuarios digitales los convierte en personas que debaten sobre los temas y opciones, los hace investigar y tomar decisiones, los convierte en "actores" políticos.

Según Vásconez (2014), en el Ecuador, los porcentajes de participación de los jóvenes, de edades comprendidas entre los 18 y 30 años, es alta, ya que corresponde a un porcentaje del $65 \%$, esto se debe en gran medida a la evolución y apropiación de estos espacios. Por ejemplo en el Ecuador, al iniciar el 2014, el dato oficial es que 7 millones de ecuatorianos tienen ya una cuenta en Facebook; ahora, a inicios del 2017, se conoce que 9 millones de ecuatorianos tienen una cuenta en esta red social, lo que significa que 9 de cada 10 personas en el Ecuador conoce e interactúa en este espacio y que "Facebook se ha convertido en la nueva primera plana de información y comunicación, ya que las noticias son compartidas y generadas por los usuarios en este medio" (C. Espinoza, comu- nicación personal, 12 de septiembre de 2014).

Precisamente hay dos investigaciones que se han realizado para determinar el grado de aceptación y uso de Facebook para temas relacionados con la política en la ciudad de Quito en Ecuador. Una de ellas -Camino a Carondelet 2017se encuentra en curso ya que aborda el tema de la campaña política para presidente del Ecuador en el año 2017; la otra investigación' se llevó a cabo en el año 2014, en época de campaña electoral para la alcaldía de la ciudad. En este estudio, la mayoría de los encuestados, el $89 \%$, manifestaron que su principal fuente de información de las propuestas de los candidatos fue Facebook, ya que "podían interactuar e informarse de las propuestas de campaña, entrevistas y actitudes de los candidatos, de manera más dinámica e inmediata y sin los filtros de los mass medias tradiconales", incluso comentaron que este espacio les permitió compartir información y propuestas específicas que ellos consideraban importantes para la ciudad.

\footnotetext{
${ }^{1}$ Trabajo de investigación realizado por Alberto Rengifo Hidalgo en el 2014 en Quito, Ecuador, en el contexto de campaña electoral de elecciones distritales para Alcalde de Quito. Basados en las Técnicas de investigación en sociedad, cultura y comunicación, de Luis Jesús Galindo Cáceres, se aplicó la metodología de encuesta a una muestra de 520 ciudadanos jóvenes entre 19 y 25 años, esta muestra se elaboró de manera aleatoria simple en donde se realizó una selección al azar de la muestra de estudio.
} 


\section{Fuente de información para Elecciones de Alcalde, Quito 2014}

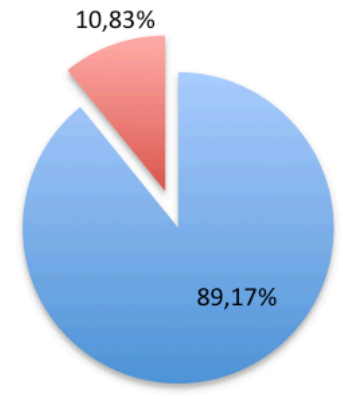

- Sí es una fuente de información

No es una fuente de información

Fig. 3. Facebook como fuente de información política.

Elaborado por Alberto Rengifo H., 2014.

Además, este trabajo reveló que existe una participación bastante activa en Facebook de los jóvenes de Quito, y esta participación abarca temas fundamentales para la sociedad como temas políticos e informativos. Esto se debe en gran medida a que Facebook es un espacio idóneo para la promoción política de un candidato o para la generación de debates con respecto a esta temática, gracias a la facilidad para acceder a la información que se presenta, ya que el poder conocer datos, propuestas e ideas sin barreras o restricciones, permite interactuar de manera directa con los candidatos o pro- tagonistas, y esto hace que los jóvenes puedan consumir la cantidad y el tipo de información que ellos deseen; por eso, se observa que los encuestados escogen a Facebook como fuente de información privilegiada para temas políticos y sociales.

Actualmente, en el marco de las elecciones para presidente de la República del Ecuador 2017-2021, se ha realizado un trabajo investigativo ${ }^{2}$-Camino a Carondelet 2017- en la ciudad de Quito, acerca del grado de aceptación y uso de Faceboook como herramienta de comunicación política y fuente de información para el electorado.

\footnotetext{
${ }^{2}$ Trabajo de investigación en curso realizado por Alberto Rengifo Hidalgo en la ciudad de Quito. Se trabaja con encuestas y grupos focales a un número de 600 ciudadanos en edades comprendidas entre 16 y 60 años. Esta muestra se elaboró de manera aleatoria simple en donde se realizó una selección al azar de la muestra de estudio.
} 
Este nuevo panorama electoral presenta a 8 candidatos que pugnan por llegar a Carondelet. Lo interesante de esta nueva campaña política es el intenso uso de las redes sociales Facebook, especialmente- como un espacio y plataforma para llegar a la ciudadanía. Los 8 candidatos tienen cuenta en Facebook -a manera de fanpage- y la utilizan constantemente para comunicarse con la ciudadanía, publicar fotos y videos de sus recorridos alrededor del Ecuador, entablar "conversaciones" en tiempo real vía Livestream, medir reacciones, receptar preguntas y sugerencias.
Varios de los candidatos tienen "programas" periódicos en Facebook, en los que abordan temas sensibles de sus propuestas. Por ejemplo, Cynthia Viteri y Mauricio Pozo, candidatos por el Partido Social Cristiano, tienen un segmento llamado CYNTHIA + MAURICIO \#CAMBIOPOSITIVO, donde han abordado temas tales como subsidios, deuda pública y comercio exterior. Por su parte Guillermo Lasso tiene un progama llamado \#YO PREGUNTO VAMOS POR EL CAMBIO, en el que se dedica a responder inquietudes ciudadanas que son propuestas vía Facebook Messenger por los simpatizantes del canditado de la alianza CREO-SUMA.

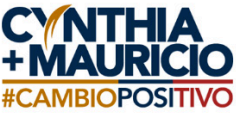

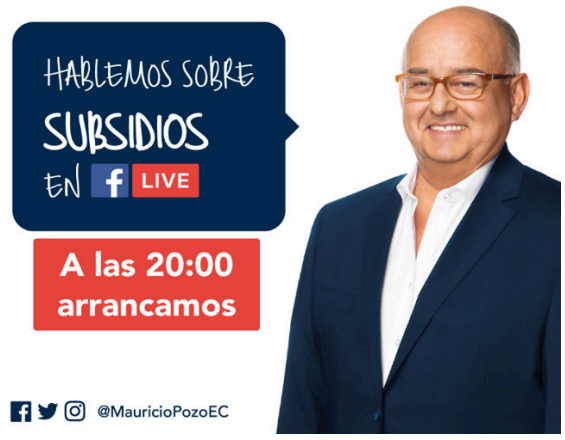

Fig. 4. Programa en Facebook Live de Cynthia Viteri y Mauricio Pozo.

Fuente: Fanpage de Cynthia Viteri y Mauricio Pozo, 2017. 


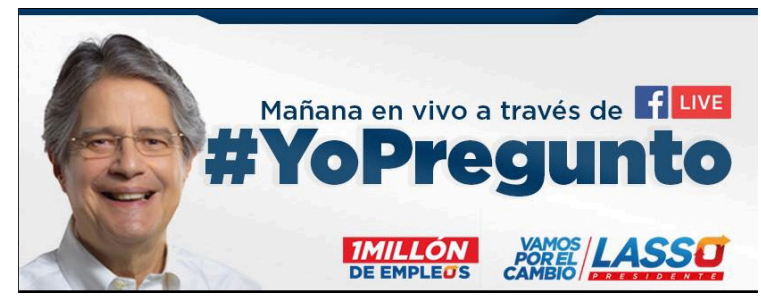

Fig. 5. Programa en Facebook Live de Guillermo Lasso.

Fuente: Fanpage de Guillermo Lasso, 2017.

Todos los candidatos a la presidencia de la República del Ecuador interactúan constantemente en sus respectivas páginas, se solidarizan con situaciones catastróficas de índole nacional e internacional, envían mensajes de aliento y positivismo al inicio de cada semana y comentan acerca de las últimas disposiciones realizadas por el gobierno central.
Los resultados que arroja la investigación Camino a Carondelet 2017 son los siguientes: el 75,3 \% del público encuestado usa Facebook hace más de cuatro años; es decir, que son usuarios antiguos y usan esta red más de una hora diaria; alrededor del $88 \%$ de los encuestados la usan como una fuente de información de los acontecimientos nacionales e internacionales.

\section{Registro en Facebook}

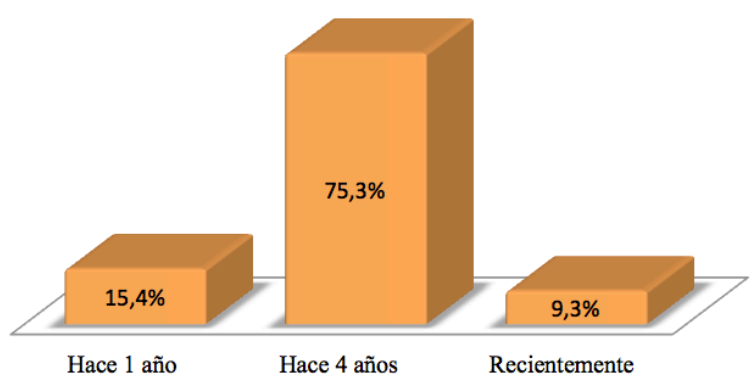

Fig. 6. Registro en Facebook TOTAL GLOBAL.

Elaborado por Alberto Rengifo H., 2017. 
Otro resultado de la investigación determina que existe un gran número de prosumidores mediáticos; es decir, ciudadanos que consumen y generan información y opinión al mismo tiempo. Alre- dedor del 65,3\% del público encuestado comentó que usa Facebook generando -compartiendo- información para que otros usuarios puedan "consumirla", transmitirla y "viralizarla".

\section{Involucramiento en Facebook}

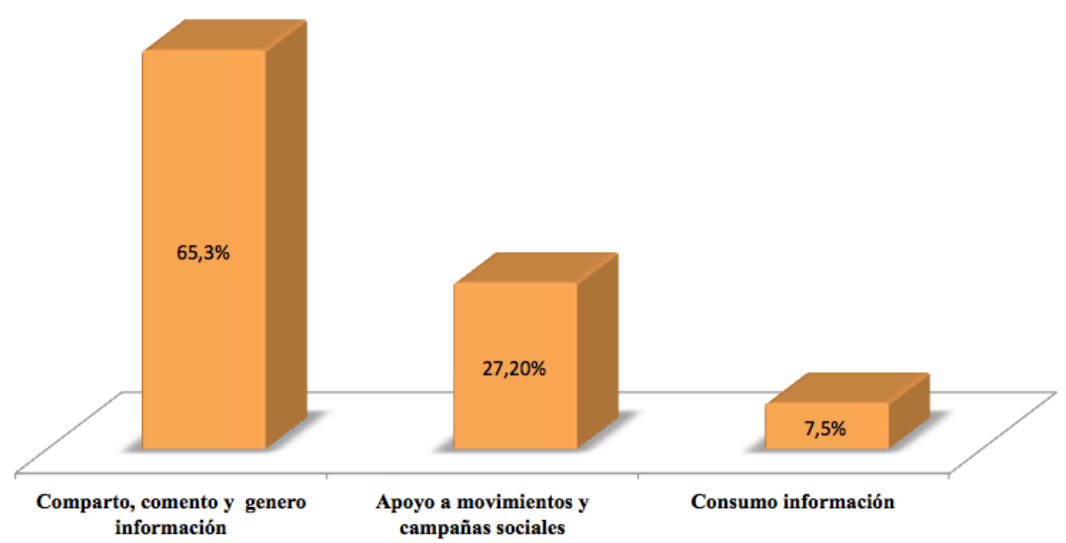

Fig. 7. Involucramiento en Facebook TOTAL GLOBAL.

Elaborado po: Alberto Rengifo H., 2017.

Si bien la investigación está en curso y es necesario llegar a la etapa final de la campaña política para poder obtener resultados concluyentes y definitivos, se podría decir que existe una par- ticipación bastante activa en Facebook por parte de los habitantes de Quito, y esta participación abarca temas fundamentales para la sociedad como temas políticos e informativos. 


\section{CONCLUSIONES}

Sin lugar a dudas, Facebook y las redes sociales han cambiado el significado de la participación social, las propias organizaciones piden la colaboración de sus ciudadanos a través de las redes para lograr de esta manera posicionarse en el marco de la opinión pública. Se ha posicionado un espacio político dentro de esta red social, tanto para los actores políticos que buscan posicionarse en el electorado, como para el electorado que busca ser prosumidor mediático en este contexto.

Gracias a estas plataformas y plazas de información, cada persona se convierte en un medio y esto obliga al político a ir a donde vaya la gente, a aprender el lenguaje y los formatos de comunicación de los usuarios, a saber cómo y qué hacer en estos espacios. Por eso, como lo sostiene Espinoza (2014), los políticos deben tener cierta formación en redes sociales, ya que un político conocido que no sepa manejarlas, puede generar una crisis política a nivel mundial. Como también un político no conocido puede posicionarse y hacer que su mensaje se viralice a una velocidad inimaginable.

Si bien el político debe "adaptarse" al público actual, los usuarios también deben poseer un nivel de conocimiento que permita usar de mejor manera estas redes. Christian Espinoza considera que para poder aprovechar al máximo estas herramientas, las nuevas generaciones deben tener una formación especializada en el tema, por eso se debe estar en proceso constante de capacitación, la gente tiene que aprender a autoeducarse; consultar fuentes, no dejarse llevar por rumores; se necesita mayor formación en el ciudadano para que pueda distinguir la información falsa de la verdadera.

Facebook es un espacio escogido especialmente por el público joven para informarse, interactuar y actuar en relación a temas políticos. Es más, el sentido de la política ha cambiado, ya que la gente tiene un espacio permanente de exigencia de resultados. Antes, esta exigencia se hacía únicamente en elecciones y a través de los medios. Ahora el ciudadano hace sus exigencias todo el día en sus espacios propios. Por eso, "la ciudadanía nunca tuvo un nivel de participación tan activa como ahora. Twitter y Facebook son un vivo ejemplo de esto, todos los días se ve a gente manifestando sus opiniones sobre cierto tema. Nunca hubo un nivel de activismo político ciudadano tan visible y alto como el de hoy. Más bien, con estas nuevas herramientas tecnológico-comunicacionales, se evita salir a las calles" (Espinoza, 2014).

Al tener la política este espacio en los medios digitales, cambia totalmente 
su concepción y manera de interacción. Para Espinoza (2014), gracias a Facebook, ya no se puede hablar de una política tradicional;"simplemente ya no se puede presentar una propuesta y esperar que esta propuesta sea concebida de una única manera, ahora debe haber más interacción con el político que busca promocionarse por redes sociales".

Como lo menciona Resina de la Fuente (2010), en una coyuntura donde los tradicionales lugares de socialización política se encuentran en proceso de quiebre, y los medios de comunicación convencionales son cuestionados por su déficit democrático en la elaboración de la agenda setting ${ }^{3}$, el espacio digital abre una nueva vía de participación que, en paralelo y en contacto con las antiguas, supone una oportunidad para la ciudadanía, en términos de activismo y deliberación sobre los asuntos públicos. Oportunidad que, sin duda alguna, constituye un nuevo espacio y un nuevo "modus vivendi" de hacer e informar sobre la política en la sociedad, especialmente en los más jóvenes. “Es que es innegable que las vidas de los adolescentes están mediatizadas porque los medios digitales son parte central de sus experiencias extraescolares y de sus relaciones e identidades cotidianas" (Buckingham, D. \& Martínez-Rodríguez, J., 2010, p. 30).
Los partidos políticos como instituciones y organismos claves en el juego democrático tienen la tarea, el desafío y la obligación de adaptarse y modelarse a los nuevos contextos. Si bien deben mantener su esencia ideológica y su praxis política, deben de igual manera "evolucionar" a la demanda actual; es decir, adecuarse a los nuevos espacios de comunicación, por donde el individuo político busca información y, sobre todo, interacción. No comprender este fundamental rol de los medios digitales sería, en palabras del sociólogo y político chileno Ricardo Núñez, conducir a los partidos y organizaciones políticas a caer en el desuso y desaparición.

"Este escenario de cambio y adaptación en el que estamos viviendo está creando una cultura participativa de las audiencias más jóvenes sin precedentes" (García-Galera, C. \& Valdivia, A., 2014, p. 11); por eso, las redes se han transformado en una herramienta eficaz para la comunicación-interacción política. En el caso de Facebook, nuestro caso de análisis, Lim (2012) manifiesta que es la "herramienta clave para organizar y coordinar movilización y acciones políticas en muchísimas ciudades alrededor del mundo" (p.62) ya que, "los usuarios la están convirtiendo en una palanca para cambiar el mundo"(Orihuela, 2008, p.58).

\footnotetext{
${ }^{3}$ Selección temática de los medios que influye en el qué y cómo entender los hechos y a los actores del
} acontecer. 
¿Se pueden generar cambios posicionamiento- desde y con las redes fundamentales desde las redes socia- sociales?, sin miedo a equivocarme diría les?, una respuesta arriesgada sería que que sí, principalmente porque la Intersí. Este cuestionamiento sería digno de net -redes sociales- "es un instrumento un análisis más profundo con otro en- fuera del tradicional control de las élites foque, por lo que yo reformularía esta y que ha permitido, principalmente a los pregunta y la plantearía de la siguiente jóvenes, ser actores capaces de incorpomanera: ¿se puede cambiar la manera rar nuevas demandas al sistema polítide ejercer y participar en temas políti- co mundial" (Morán, M. \& Benedicto, J., cos -elecciones, consultas, referendos, 2008, 152). 


\section{BIBLIOGRAFÎA}

Acosta, G., Maya, C. (2012). Participación política en redes sociales: el caso de los grupos en Facebook. Colombia: Sello Editorial Universidad de Medellín. Recuperado de http://www.scielo.org.co/ scielo.php?script=sci_arttext\&pi$\mathrm{d}=$ S0120-48232013000200011

Buckingham, D., \& Martínez-Rodríguez, J., (2013). Jóvenes Interactivos: Nueva Ciudadanía entre redes sociales y escenarios escolares. Barcelona, España: Grupo Comunicar.

Castells, M. (2007). La transición a la sociedad red. Barcelona, España: Ariel.

García-Galera, C. \& Valdivia, A., (2014). Prosumidores mediáticos. Cultura participativa de audiencias y responsabilidad de los medios. Huelva, España: Grupo Comunicar.

Gutiérrez Rubí, A., (2014). Tecnopolítica. España: Grafiko

Igarza, R., (2009). Burbujas de ocio. Buenos Aires, Argentina: Icrj inclusiones.

Lim, M., (2012). Clicks, Cabs, and Coffe Houses: Social Media and Oppositional Movements in Egypt, 20042011. Arizona, Estados Unidos, Journal of Communication.

Martínez, J. G., Fortuño, M. L., \& Vidal, C. E. (2016). Las redes sociales y la educación superior: las actitudes de los estudiantes universitarios hacia el uso educativo de las redes sociales, de nuevo a examen. Cataluña, España: Universidad Rovira i Virgili. Recuperado de http://search.proquest.com/docview/1820542501/ fulltextPDF/1F7716A065A84941PQ/1?accountid $=13250$

McLuhan, M. (1994). Comprender los medios de comunicación-Las extensiones del ser humano. Buenos Aires, Argentina: Ediciones Paidós Ibérica S.A. Recuperado de http://cedoc.infd.edu.ar/upload/ McLuhan_Marshall de_comunicacion.pdf

Morán, M. \& Benedicto, J., (2008). Los jóvenes como actores sociales y políticos en la sociedad global" en Pensamiento Iberoamericano. Madrid, España: Universidad Complutense de Madrid y UNED.

Observatorio Nacional de las Telecomunicaciones y de la SI (ONTSI). (2012). Estudio sobre el conocimiento y uso de las redes sociales. España: Ontsi.red.es. Recuperado de http://www.ontsi.red.es/ontsi/ sites/default/files/redes_socialesdocumento_0.pdf

Orihuela, J., (2008). Internet: la hora de las redes sociales. Navarra, España: Facultad de Comunicación de la Universidad de Navarra-Nueva 
Revista de Política, Cultura y Arte. Resina de la Fuente, J., (2010). Redes sociales, ciberpolítica y nuevas movilizaciones: el impacto digital en los procesos de deliberación y participación ciudadana. Madrid, España: Universidad Complutense de Madrid.

Savigny, H., (2002). Public opinion, political communication and the Internet. Birmingham, United Kingdom: University of Birmingham.

Scherman, A.; Arriagada, A. (2010). ¿Ciudadanía Digital, Participación Tradicional? Jóvenes, Participación Política y Consumo de Medios en Chile. Chile: Universidad Diego Portales. Recuperado de www. udp.cl/funciones/descargaArchivos.asp? seccion=documentos\&id=135

Svampa, M., (2008). Cambio de época. Movimientos sociales y Poder Político. Buenos Aires, Argentina: Siglo veintiuno.

Tascón, M., \& Quintana, Y., (2012). Ciberactivismo. Las nuevas revoluciones de las multitudes conectadas. Madrid, España: Catarata.

Thompson J, (1998). Los media y la modernidad. Una teoría de los medios de comunicación. Barcelona, España: Ediciones Paidós Ibérica, S.A. 
